\title{
Bericht.
}

\section{Sammelbericht über die im Jahre 1904 in Rumänien veröffentlichten Arbeiten gynäkologischen und geburtshülflichen Inhalts.}

\author{
Von \\ Dr. E. TOFF, \\ Frauenarzt in Braila.
}

1. Papanicol, Die Retention des nachfolgenden Kopfes in der Gebärmutter. Revista de chirurgie. 1904. No. 1. S. 1.

2. Botescu, Orarialcystc mit Stieldrehung. Societatea de chirurgic in Bukarest. Sitzung vom 5. XI. 1903.

3. Toff, E., Über die Prophylaxis der puerperalen Infektionen. România medicala. 1904. No. 8. p. 179.

4. Demetrian, C., Hydatidenmole. Revista de chirurgie. 1904. No. 2. p. 67.

5. Bogdan, G., Ein neuer Fall von Stur\%geburt, gefolgt von Abreissung der Nabelschnur und Fallen des Kindes. România medicala. 1904. No. 9. p. 208.

6. Manolescu, Alex., Die Sterblichkeit der kleinen Kinder. România medicala. 1904. No. 9. p. 208.

7. Vasiliu und Negruzzi, Schwangerschaft lompliziert mit Orarialcyste. Puerperalfieber. Rerista de chirurgie. 1904. No. 3. p. 120.

8. Ionescu, Th., Totale abdominale Hysterektomie mit ileolumbaler Beckenausrāumung wegen Krebs des Collum uteri. Societatea de chirurgie in Bukarest. Sitzung vom 19. XI. 1903.

9. Babes, V., Diffuses Carzinom des Uterus. Societatea anatomica in Bukarest. Sitzung rom 20. XII. 1903.

10. Cealâc, Symmetrisches Sarkom der Orarien. Societatea anatomica. Sitzung rom 24. 1. 1904.

11. Ionescu, Th., Primitires Epitheliom des rechten Orariums, doppelscitige Salpingitis. Totale abdominale Kastration, Durchschneidung des rechten Ureters, Naht beider Stūcke. Heilung. Socictatca do chirurgie in Bukarest. Sitzung vom 26. I. 1y04.

12. Bonachi, Torsionierte Himatosalpinx. Societatea de chirurgie in Bukarest. Sitzung vom 4. II. 1904.

13. Alexandrescu, Die Assistenz der Wöclinerinnen auf dem Lande. Spitalul. 1904. No. $12 / 13$. p. 367.

14. Gheorghiu, Puerperale interstitiale Uterinabszesse. Revista de chirargie. 1904. No. 5. p. 208.

15. Zambilovici, Vollstāndige uterine Inversion mit adhärenter Placenta. Revistal de chirurgie. 1904. No. 5. p. 217.

16. Ionescu, Th., Doppelte, eitrige, ins Rectum durchgebrochene Salpingitis. Totale abdominale Hysterektomie. Societatea de chirurgie in Bukarest. Sitzung vom 14. IV. 1904.

17. Cealâc, M., Einige Worte ūber das letale Ende bei Uteruskrebs. Revista de chirurgie. 1904. No. 7. p. 328. 
18. Racoviceanu und Bogdanovici, Doppelte extra-uterine Schwangerschaft, uterine Schwangerschaft mit fötaler Retention. Chirurgische Gesellschaft in Bukarest. Sitzung rom 28. IV. 1904.

19. Cealâc, Durchbohrung der rekto-vaginalen Wand mit Riss des Perineums, infolge eines brutalen Coitus. Revista de chirurgie. 1904. No. 6.

20. Cohn, Kongenitale Cyste der Vagina. Societatea de chirurgie in Bukarest. Sitzung vom 26. V. 1904.

21. Cristeanu, Hysterektomie und akute Puerperalinfektion. Revistil de chirurgie. 1904. No. $8 / 9$. p. 338.

1. Papanicol. Nach einer historischen Übersicht der Frage gibt $P$. die Beschreibung von zwei selbst beobachteten Fällen, in welchen der nachfolgende Kopf sich vom Rumpfe trennte und gesondert extrahiert werden musste.

Fall I. Die 26jährige Primipara befand sich im fünften Monate der Gravidität, als sie von Geburtswehen überrascht wurde. Der Fötus wurde mit vorangehenden Füssen expulsiert und von der assistierenden Hebamme mit solcher Kraft gezogen, dass der Kopf sich vom Rumpfe trennte und in der Uterushöhle verblieb. Bei der Untersuchung wurde die Gebärmutter fast vollstiindig geschlossen gefunden, derart, dass nur mit grosser Mühe der Zeigefinger eingeführt werden konnte, er aber keineswegs imstande war, den zurückgehaltenen Schädel zu extrahieren. Erst nach vorgenommener Perforierung mit einer dünnen Sonde und Erweiterung dieser Öffnung konnten teilweise die Schädelknochen, dann das Encephalum mit dem Finger extrahiert werden, wobei es sich herausstellte, dass noch ein Fötus vorhanden war, welcher quer lag und durch Wendung hervorgeholt wurde. Am folgenden Tage wurde die zurückgebliebene Placenta manuell extrahiert. Leichtes Fieber bis $38^{\circ}$ in den ersten $z$ wei Tagen, sonst verlief das Wochenbett normal.

Fall II. Die 25jälırige IIpara, im vierten Monate der Gravidität, hatte ohne Assisten\% entbunden, dabei löste sich der Kopf ab und blieb in Cavum uteri. Am zweiten Tage wurde dieselbe ins Krankenhaus gebracht, und als die Vorbereitungen zur Extraktion getroffen wurden, erfolgte eine uterine Kontraktion, und der Kopf wurde ausgestossen.

P. ist der Ansicht, dass in allen Fällen ron Kopfretention die Entfernung möglichst bald erfolgen soll, da eine exspektative Behandlung nicht ohne Gefahr über einige Stunden ausgedehnt werden kann. Als Vorzugsmethode ist die digitale Ausräumung anzusehen, eventuell unter Anwendung eines Metreurynters.

2. Botescu. Der Fall bot die Eigentümliclıeit, dass die Patientin nach einer Geburt war und mit der Diagnose Puerperal- 
fiel,er ins Krankenhaus geschickt worden war. Man konstatierte ausser Fieber auch einen Abdominaltumor, und als die eingeleitete Behandlung den Zustand besserte, wurde die Laparotomie ausgeführt und eine Ovarialcyste mit gedrehtem Stiele gefunden und entfernt. Heilung.

3. Toff. Trotzdem das Wesen und die Prophylaxis des Wochenbettfiebers genügend gut bekannt sind, sterben doch noch zahlreiche Friuen an puerperalen Infektionen. Der Grund ist hauptsächlich in der mangelhaften Schulung und in der Nachlässigkeit der Hebammen zu suchen. T. schlagt vor, den Hebammen das Tragen von Gummihandschulen bei der Untersuchung Schwangerer und während der Assistenz bei der Geburt obligatorisch vorzuschreiben. Das Touchieren soll auf das minimalste beschränkt werden und die Diagnose intra partum mehr durch iussere Handgriffe, Palpation, Beckenmessung und Auskultation gestellt werden. Der Unfug, die Gebärende unzählige Male ohne Zweck und Nutzen vaginal zu untersuchen, soll aufhören. Für jede Wöchnerin soll die Hebamme einen Temperatur- und Pulsbogen anlegen, mit der Verpflichtung, allsogleich ärztliche Hülfe zu verlangen, wenn die T'emperatur das Normale übersteigt, oder, was eben so wichtig ist, der Puls frequent wird und 90 übersteigt. Durch recht\%eitiges Eingreifen kann oft schweren Infektionen vorgebeugt werden. Auch eine genaue Untersuchung der Nachgeburt gehört zu den wichtigen prophylaktischen Massnahmen, da in den meisten Fällen ron Puerperalinfektion Placentarreste im Gebärmuttercavum gefunden werden. Endlich sollen die Hebammen mit der Bereitung steriler Lösungen und mit der Benützung der Antiseptika viel melır vertraut gemacht werden, als es bis nun der Fall gewesen ist. Behufs Vornalume ron Vaginalwaschungen soll jede Wöchnerin ihr eigene Glaskanüle haben.

4. Demetrian. Die betreffende 21 jährige Patientin befand sich in dritten Monate der Schwangerschaft, Latte fast rom Beginne derselben an Vaginalblutungen gelitten, wïhrend die Gebärmutter sich rasch vergrössert hatte und fast die Grösse einer im achten Monate der Graviditiit befindlichen erreicht hatte. Es bestand bedeutende Anïmie, Ödem an den Knöcheln, Dyspnoe, Puls 130, Pemperatur $38,5^{\circ}$, ausserdem Eiweiss im Harne. In Anbetracht der grossen Schwäche wurde die Entleerung der Gebärmutterhöhle beschlossen, zuerst ein Ballon ron Champetier de libes eingelegt und nich genügender Erweiterung alles digital ausgeriumt. Trotz allen entsprechenden Jassnahmen konnte der Kräfteverfall der Kranken nicht aufgehalten werden 
und erlag dieselbe 10 Tage nach dem Eingriffe. Bei der Nekropsie wurde die Uterusschleimhaut fetzig, mit tiefen Divertikeln, die Muskularis dünn, blass und an einigen Stellen wie fehlend gefunden. Verfasser hebt hervor, dass in einem ähnlichen Falle die Erwägung einer abdominalen Hysterektomie, wie diesclbe von Fournel empfohlen wurde, volle Berechtigung hat, da eine restitutio ad integrum nicht zu erwarten gewesen wäre.

5. Bogdan. Derartige Fälle kommen selten ror, so dass in der Literatur nur 3 verzeichnet sind: einer von Tissier und zwei von Budin. Der von B. beobachtete Fall betraf eine 20jülırige IIpara, welche stehend entbunden hatte, und geschah die Ausstossung des Kincles mit einer solchen Schnelligkeit, dass dasselbe nicht aufgehalten werden konnte und auf den Boden fiel, ohne sich aber Verletzungen zuzuziehen; hierbei riss die Nabelschnur von ihrer Insertionsstelle am Nabel ab, und eine reichliche Blutung fand statt, welche durch Anlegung einer Klemmzange gestillt wurde.

6. Manolescu. Verfasser weist auf die grosse Sterblichkeit der Kinder in zartem Alter in Rumänien hin. Die Ursache ist hauptsächlich in der mangelhaften Pflege, in der schlechten Ernährung und in den zahlreichen Nabelschnurinfektionen zu suchen. So gaben z. B. in einem Bezirke die Kinder in Alter bis zu sechs Monaten 21 pCt. der Gesamtmortalitit, in einem anderen Bezirke sogar $24 \mathrm{pCt}$., während die Sterblichkeit von 6 Monaten bis zu $1 \mathrm{Jahr}$ nur 6 pCt., bezw. nur 4 pCt. betrug. Der Mangel an guten Hebammen auf dem flachen Lande trägt einen grossen T'eil der Schuld an diesen Zuständen, da die Assistenz bei den Geburten meist von alten Weibern geleistet wird, deren empirische Kenntnisse sehr mangelhafte sind.

7. Vasiliu und Negruzzi. Die Operation der Orarialcysten ist heute in der Chirurgie der Abdominalorgane ein banaler Eingriff, solange keine besonderen Konplikationen da\%ischentreten, keine ausgedehnten Verwachsungen bestehen und die Asepsie gewahrt werden kann. Gan\% anders stellt sich der von den Verfassern beschriebene Fall dar. I)ie 19jährige Patientin litt bereits seit 2 Jahren an einer rechtsseitigen Ovarialcyste, als sie niederkan und an Puerperalfieber erkrankte. Trotz vorgenommener Auskratzung des Uteruscavums', wobei zahlreiche fötide Membranfetzen entfernt wurden, blieb die Temperatur hoch, die Pulsfrequenz über 120, und war der Allgemeinzustand ein sehr schwerer. Man nahm eine komplikatorische Eiterung von seiten der Eierstockcyste an und schritt zur Entfernung derselben. Tat- 
sïchlich wurde eine multilokuläre Cyste mit torsioniertem Stiele gefunden, deren einige $\mathbf{A}$ bteilungen mit eitriger Flüssigkeit erfüllt waren, während andere einen dickflüssigen, trüben, rotbraunen Inhalt aufwiesen. Die zahlreichen, sehr festen Adhärenzen erschwerten ausserordentlich die Ablösung des Neugebildes, namentlich vom Coecum, mit welchem dasselbe breit verwachsen war, ausserdem riss der eiterhaltige Sack während der $A$ blösungsarbeit ein, und die Flüssigkeit entleerte sich in die freie Bauchhöhle. Zwar wurde dieselbe mit sterilen Kompressen und künstlichem Serum entfernt, doch wurde vorsichtshalber eine Mikuliczsche Drainierung angelegt. Die Folgen der Operation waren über alle Erwartungen günstige, denn die Kranke erholte sich rasch, das Fieber sank, und sie konnte nach $2^{1 /}$. Monaten das Krankenhaus geheilt verlassen.

10. Cealâc. Die Krankheit der 25jährigen, früher immer gesund gewesenen Patientin hatte vor 2 Nonaten begonnen und war der Status praesens folgender: Es bestand grosse Schwäche ausgesprochene Kachexie, die Hautdecken hatten ein fahles, erdiges Aussehen, der Bauch war bedeutend ausgedehnt, enthielt freie Flüssigkeit, und es hatte sich auf den Bauchdecken Kollateralkreislauf entwickelt. Fast seit Beginn der Erkrankung waren Schmerzen in der linken Fossa iliaca, die immer heftiger wurden, aufgetreten. Die vorgenommene Paracentese förderte 7 Liter Flüssigkeit zu Tage und konnte nun durch die kombinierte Untersuchung festgestellt werden, dass beiderseits in den Adnexen je ein orangengrosser Tumor vorhanden war. Die von Kiriak vorgenommene Laparotomie zeigte beide Ovarien von je einer nierenförmigen Neubildung eingenommen, welche sich mikroskopisch als Rundzellensarkom erwies, ähnlich den von Le opold und M a rchand beschriebenen gemischten Tumoren, welche sowohl den sarkomatösen, als auch den krebsigen Geschwülsten angehören. Dieselben zeichnen sich durch ihre ausserordentliche Malignität aus. Das Resultat der Operation war infolge der ausserordentlichen Schwäche der Patientin, ein fatales.

12. Bonachi. Es bestand gleichzeitig eine Schwangerschaft im dritten Monat, und da man den faustgrossen Tumor in der linken Darmbeingrube für eine Hydronephrose, beruhend auf Ureterenkompression durch den schwangeren Uterus, hielt, wurde die Einleitung des künstlichen Abortus beschlossen. Tatsüchlich besserten sich die schweren Symptome, welche bis dahin bestanden hatten, wie unstillbares Erbrechen, heftige, in das linke Bein ausstrahlende Schmerzen etc. für einige Tage, doch traten die- 
selben wieder auf, und es erfolgte Exitus lethalis. Bei der Sektion wurde eine allgemeine Peritonitis, hervorgerufen durch die Perforierung eines eitrigen Sackes der linken Andnexen, gefunden. Nach der Beschreibung scheint es sich also nicht um Hämotosalpinx, sondern um Pyosalpinx gehandelt zu haben.

14. Gheorghiu. Die betreffende 22jährige Frau hatte eine normale Geburt und ein normales Wochenbett durchgemacht, als sie 14 Tage später etwas febrile Temperatur zeigte. Es wurden intrauterine Einspritzungen vorgenommen, doch schwankte das Thermometer zwischen $37^{\circ}$ und $37,4^{\circ}$, während lokal, ausser einer Verdickung der vorderen Muttermundslippe, nichts Abnormes beobachtet werden konnte. Der Zustand dauerte mit Remissionen und Exazerbationen etwa 12 Tage und entleerte sich dann im Laufe einer intrauterinen Spülung, beim Ziehen an der vorderen Muttermundslippe mittels Hackenzange, aus derselben plötzlich 7-10 g Eiter, welcher einer in der Uteruswand befindlichen Höhle entstammte. Die Öffnung wurde etwas erweitert, ausgespült und heilte in wenigen Tagen, worauf auch die Temperatur zur Norm zurückkehrte.

Uns will es scheinen, dass es sich in diesem Falle eher um eine lokale Infektion durch ein früheres Anfassen mit der Hackenzange, durch den untersuchenden Finger oder die Irrigatorkanüle, als um einen wahren interstitialen Abzess der Uteruswand gehandelt hatte.

15. Zambilovici. Die 28jährige Ypara wurde von einem alten Weibe entbunden, welches unmittelbar nach der Geburt an der Nabelschnur zog, wodurch sowohl eire vollstiinlige Inversion der Gebärmutter samt der adhärenten Placenta, als auch ein Abreissen der Nabelschnur an der placentaren Insertionsstelle bewirkt wurde. Die Ablösung des Mutterkuchens konnte wegen der gleich einsetzenden bedrohlichen Blatung im vorgefallenen Zustande nicht gemacht werden. In den folgenden Tagen waren febrile Temperaturen bis $39,6^{\circ}$ und übelriechende Lochien zu verzeichnen, welche auf Spülungen mit künstlichem Serum zurückgingen.

16. Jonescu. Die rektale Durchbruchsöffnung liess den Zeigefinger durch. Bei der vorgenommenen totalen Hysterektomie konnte dieselbe nicht geschlossen werden, da zahlreiche Adhärenzen und namentlich das harte, sklerosierte Gewebe in der Umgebung dies verhinderte, infolgedessen beschränkte sich J. darauf, die Peritoneallı̈̆hle durch Einnähen des Kolon abzuschliessen unà durch die Vagina zu drainieren. Die Folgen waren gute, cla die Fistel 
sich von selbst schloss und Patientin geheilt entlassen werden kounte.

17. Cealâc. Der Verfasser beschreibt das Endstadium und die Nekropsie von zwei an Gebärnutterepitheliom verstorbenen Patientinnen als Beitrag zur Beantwortung der Frage über die eigentliche Todesursache bei dieser Krankheit. Es stellt sich heraus, dass dieselbe auf einer Nierenerkrankung mit konsekutiver Urïmie, hervorgerufen durch eine Kompression der Ureteren, beruht. In einem der beobachteten Fälle waren die Ureteren oberhalb der durch die Krebsmassen komprimierten Stelle bis z.11' Dicke einer Darmschlinge dilatiert, das Nierenbecken war faustgross, das Gewebe der Niere sehr verringert und von zahlreichen kleinen Cysten durchsetzt. Die betreffende Kranke hatte immer weniger und schliesslich nur etwa $100 \mathrm{~g}$ tïglich uriniert, der Harn war einweisshaltig, es stellten sich exquisit urämische Symptome ein, denen Patientin schliesslich auch erlag. Auch im zweiten Falle war der Verlauf ein ähnlicher und bei der Sektion wurden stirk erweiterte Harnleiter, eine grosse weisse und eine kleine weisse Niere gefunden.

18. Racoviceanu und Bogdanovici. Die 24jalhrige Patientin hatte vor 5 Jahren normal geboren, wurde einige Monate später wieder schwanger, fühlte die Kindsbewegungen, und im achten Monaten traten Geburtswehen auf, welche 36 Stunden dauerten, aber das Kind kam nicht zum Vorscheine, der Tumor blieb, während alle übrigen Erscheinungen verschwanden. Seit \%wei Jahren ist diese, in der rechten Weiche befindliche Geschwulst erheblich kleiner geworden. Zwei Monate nach Beendigung dieser Pseudogeburt zeigten sich wieder normale Menstruationen, welche während einiger Monate wiederkehrten, dann wieder Schwangerschaftssymptome, am normalen T'ermine Geburtswehen, doch expulsierte sie auch diesmal nichts. Der Tumor begann wieder ab\%unehmen, während sich aus dem Gebärmutterhalse eine dickliche olivengrüne Flüssigkeit entleerte. Die Hysterometrie ergab $14 \mathrm{~cm}$.

Die vorgenommene Laparotomie ergab folgenden merk würdigen Befund. Es wurde ein altes Lithopïdion gefunden, welches mächtige Adhärenzen mit der Gebärmutter, dem Mesenterium, dem Epiploon, den Gedärmen und der Beckenwand eingegangen wal, so dass die Exstirpation bedeutende Schwierigkeiten darbot. Der Tumor enthielt einen \%usammengeschrumpften, kleinen, sonst aber gut erhaltenen Fütus und im oberen T'eile einen zweiten Fötus, dessen Skelett sehr deutlich zu unterscheiden war. Ausser- 
dem fand man im Uterus eine normale Schwangerschaft, doch waren nur der Fundus und die hintere Wand wie bei Schwangerschaft entwickelt, während die übrige Wandung rigid geblieben war. Dies wahrscheinlich infolge der Behinderang durch das Lithopädion. 1)ie Verfasser fragen sich, ob die beiden extrauterinen Schwangerschaften simultan sind oder sich nach einander gebildet haben. Die Patientin hat, obwohl herzkrank, die Operation gut iiberstanden.

19. Cealâc. Man fand bei der 22jährigen, schwächlich gebauten Patientin eine Llzeration am Frenulum, die sich auf $\checkmark$ agina und Perineum erstreckte und den Eingang in einen Kanal dirrstellte, welcher in das Rectum führte. Die Breite dieser Öffnung war $3 \mathrm{~cm}$, falls man dieselbe mit dem Finger oder einem Speculum auseinanderzog, doch traten durch dieselbe beim Stuhlgange keine fäkalen Massen aus dem Mastdarme. An den Genitalorganen war sonst nichts Abnormes zu bemerken. Patientin gab an, diese Verletzungen bei der Entjungferung erlitten zu haben, wobei sie sehr viel Blut verloren hatte. C. konnte auch den betreffenden Mann untersuchen, fand einen abnorm grossen Penis, und erzählte derselbe, in der Brautnacht drei fruchtlose Versuche gemacht zu haben, während beim vierten die in Rede stehende Verletzung erfolgte.

Die von Kiriak anfangs vorgenommene Operation, bestehend in Ablösung der vaginalen und rektalen $W$ and un die Fistelöffnung herum und Nabt der blutenden Flächen, blieb erfolglos, auch ein zweiter Eingriff bewirkte nicht die Schliessung der Fistel und wurde endlich durch einen queren Schnitt das Perineum breit eröffnet, die Scheidenwand von dem Mastdarme in grösserer Ausdehnung abgelöst und dann durch Katgutnähte die einzelnen T'eile vereinigt. Diesmal erfolgte Heilung per primam.

21. Cristeanu. Der Verfasser macht eine ausführliche historische und kritische Studie dieser Frage und fügt den von anderen Autoren veröffentlichen Krankengeschichten noch sechs eigener Beobachtung hinzu, die aber alle tödlich endeten. Er spricht die Ansicht aus, dass die Entfernung der Gebärmutter wegen akuter Puerperalinfektion in manchen Fällen indiziert sein könnte, dass man aber im grossen und ganzen sagen muss, dass bei eigentlicher Infektion, ohne nachweisbare Lokalisierung, ron Erfolgen dieser Operation nicht die Rede sein kann und dieselbe also kontraindiziert erscheint.

Iie Indikationen, welche die Verfechter der Operation für dieselbe anführen, sind folgende: 1. Diejenigen, welche auf der 
bakteriologischen Untersuchung des Blutes beruhen. In dieser Beziehung kann gesagt werden, dass weder die Anwesenheit, noch der Mangel der Streptokokken in Blute einen Fingerzeig für die Schwere des Falles abgeben, da viele Fülle, sowohl bei Puerperalinfektion, als auch bei anderen akut infektiösen Krankheiten in Genesung übergehen, trotzdem Streptokokken im Blute gefunden werden, während andere zugrunde gehen, ohne dass ein solcher Nachweis möglich gewesen wïre. Viel besseren Aufschluss gibt die leukozytïre Formel des Blutes, insofern eine müssige Polynukleose und deutliche Eosinophilie auf eine heilsane Reaktion des Organismus hinweist und ein Fingerzeig ist, dass derselbe gegen dic Krankheit genügend kräftig reagiert, während das Ausbleiben dieser Erscheinungen auf eineu schlechten Ausgang gefasst machen muss und also durch eine Operation der tödliche Ausgang, durch eine allgemeine Schwächung des Organismus, nur beschleunigt werden kann. 2. Auch die Indikationen, welche aus der bakteriologischen Untersuchung der Uterinsekretion ge\%ogen werden, sind nicht stichhaltig, $d a$ es sich nicht immer um dieselbe Mikrobenart handelt, auch die Virulenz derselben nicht immer die gleiche ist, sondern wahrscheinlich durch die Einwirkung mehrerer Bakterienarten gegenseitig gesteigert wird.

Die Indikationen, welche für die Operation aus der Subinvolution des Uterus, der Frequenz des Pulses, der Temperaturhöhe und lem Allgemeinzustande gezogen werden, sind ebenso wenig massgebend, und es zeigen zahlreiche Beispiele, dass man aus denselben absolute Anzeigen für einen operativen Eingriff nicht ziehen kann.

\section{Dr. F. H. Kumpl $f$.}

Als die sogen. Thure-Brandtsche Methode für die Behandlung gynākologischer Leiden, spez. auf Grund der Empfehlung von Autoritäten, wie jener B. S. Schultzes, in Aufschwung kam, machte sich, wie so oft bei Neuerungen in therapeutischer Richtung, ein kritikloser Optimismus geltend, der das unzweifelhaft Gute an der Sache in den Augen nüchterner Beobachter ebenso schädigt als die Qualität der zahlreichen Apostel, welche sich für berufen bielten, diese Behandlungsart als die cinzig richtige und für alles geeignete zu proklamieren. Um all jene Charlatanerie und Überschwenglichkeit welche sich im Beginne der Massage-Ära in so reichem Masse und zum Nachteil der Richtung cntwickelt hatte, mit Erfolg auszuschalten, bedurfte es einer anderen Kategorie von Persönlichkeiten, welche durch ibre Qualităten in den Stand gesetzt waren, die Leistungsfähigkeit der Methode für eine be- 